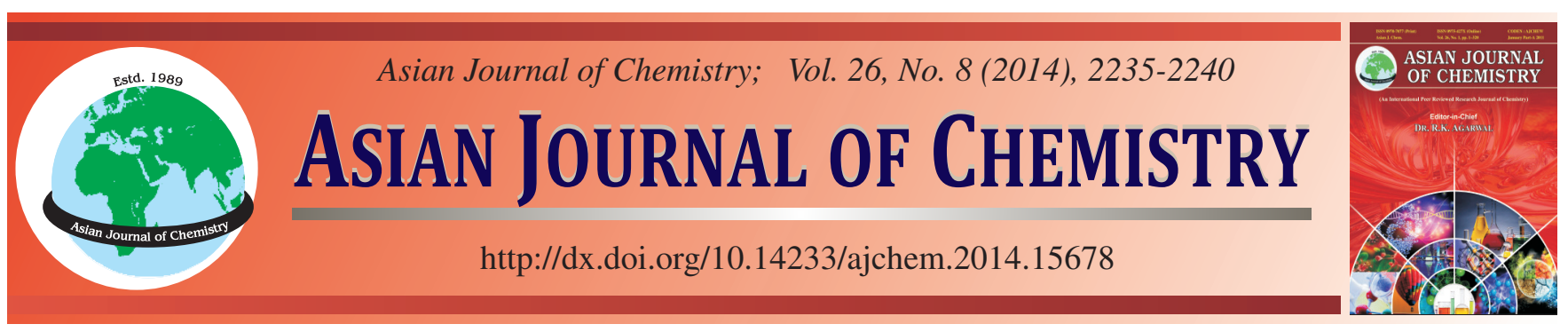

\title{
Synthesis of Cu-Doped Ti-Containing MCM-41 and its Photocatalytic Performance for Indoor Air Purification
}

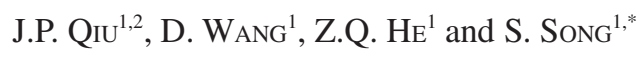

${ }^{1}$ College of Biological and Environmental Engineering, Zhejiang University of Technology, Hangzhou 310032, P.R. China

${ }^{2}$ College of pharmacy \& Materials Engineering, Jinhua College of Vocation and Technology, Jinhua 321007, P.R. China

*Corresponding author: Tel./Fax: + 86571 88320726; E-mail: ss@ zjut.edu.cn

\section{INTRODUCTION}

Nowadays, indoor air quality remains a big challenge to human health protection. Human exposure to indoor air pollutants, even a few ppm, will elevate potential health risks ${ }^{1,2}$. Therefore, great efforts have conducted to satisfy the more and more strict environmental regulations.

Presently, using air purifiers is first available solution to improve indoor air quality. Traditional air purifiers often use sorption materials to adsorb gases or odors. These purifiers only transfer the organic pollutants from one phase to another and subsequent treatments are still required to further eliminate them. Besides, adsorbents need regular replacement because of their limited capacities. Thus, people turn to investigate more efficient and environmental friendly methods. Photocatalytic oxidation (PCO) is emerging as the most promising technology to remove indoor air pollutants especially volatile organic compounds (VOCs). Photocatalytic oxidation using $\mathrm{TiO}_{2}$ as catalyst in conjunction with UV irradiation has widely studied and proven to be an effective means of controlling volatile organic compounds ${ }^{3-7}$. When $\mathrm{TiO}_{2}$ is UV-activated, it could yield a series of highly reactive electron-hole pairs, followed by hydroxyl radicals and super-oxide ions. Those substances which possess a high redox potential would further oxidize volatile organic compounds to $\mathrm{CO}_{2}$ and $\mathrm{H}_{2} \mathrm{O}$. Apparently, rather than transferring pollutants from the gas phase to the solid phase, photocatalytic oxidation can thoroughly remove them without any subsequent treatments.

However, the reaction process of the gas-solid heterogeneous photocatalysis is considerably slow because of the low contact efficiency between $\mathrm{TiO}_{2}$ and pollutants in ambient temperature and pressure. It is believed that if one can improve the adsorption ability of $\mathrm{TiO}_{2}$ towards volatile organic compounds, the removal efficiency of whole system will be promoted. Many past researches have shown that activated carbon was a good alternative to increase the pollutant concentration around $\mathrm{TiO}_{2}$. However, the adsorption efficiency of activated carbon is not very high. In this paper, we chose another adsorbent-MCM41 to support $\mathrm{TiO}_{2}$. This kind of materials possesses a hydrophobic surface with high specific surface area of approximately $1000 \mathrm{~m}^{2} / \mathrm{g}$. As a result, it was used as an adsorbent of various pollutants ${ }^{8-11}$. Besides, its adjustable surface property facilitates the dispersion of $\mathrm{TiO}_{2}$. Moreover, to enhance the catalytic activity of $\mathrm{TiO}_{2}$, many modified treatments have been done. Among them, doping metallic element aroused great interest. It is said that the doping not only changes the photocatalytic redox process but also hinders the recombination of photogenerated $\mathrm{e}^{-}-\mathrm{h}^{+}$pairs to increase the photocatalytic activity ${ }^{12}$.

In present work, MCM-41 was synthesized at room temperature to behave as adsorbent and catalyst. One transition metal, 
copper, was introduced into MCM-41 by in situ method. After incorporating $\mathrm{TiO}_{2}$ by immersion, the resulting material was used in photocatalytic test to evaluate the impact of MCM-41 and copper on the catalytic activity of $\mathrm{TiO}_{2}$. Some studies have reported the performance of Ti/MCM-41 in photocatalysis of indoor volatile organic compounds but few of them concerned about the effect of copper. A common indoor volatile organic compound, formaldehyde, was chosen as target pollutant. The adsorption capacity of various samples for it was considered. Moreover, the physicochemical property of catalyst was also discussed.

\section{EXPERIMENTAL}

Copper nitrate trihydrate and tetrabutyl titanate $\left[\mathrm{Ti}(\mathrm{OBu})_{4}\right]$ was procured from Sinopharm Chemical Reagent Co., Ltd. Tetraethyl orthosilicate (TEOS) and cetyltrimethyl ammonium bromide (CTAB) were procured from Changshu Yanghu Chemical Co., Ltd. Ammonia were obtained from Pinghu Chemical Reagent Factory. Formaldehyde was supplied by Sangon Biotech (Shanghai) Co., Ltd. All chemicals are of analytical-grade and without further purification.

Sample preparation: The preparation of $\mathrm{Cu} / \mathrm{MCM}-41$ was following Xia et al. ${ }^{13}$. Specifically, $0.9 \mathrm{~g}$ of CTAB was dissolved in $60 \mathrm{~mL}$ of absolute ethanol and stirred for $0.5 \mathrm{~h}$. Then, a certain mount of $\mathrm{Cu}\left(\mathrm{NO}_{3}\right)_{2} \cdot 3 \mathrm{H}_{2} \mathrm{O}$ were added in the CTAB solution. After stirring for $0.5 \mathrm{~h}, 80 \mathrm{~mL}$ of deionized water was added into mixed solution followed by $2 \mathrm{~mL}$ of TEOS. After stirring for $1 \mathrm{~h}, 3.4 \mathrm{~mL}$ ammonia (25 wt. \%) was added to the mixed system dropwise to form a sol. The synthesis media was kept under vigorous stirring condition for $9 \mathrm{~h}$. The whole procedure was conducted at room temperature. The products were filtered, washed and dried at $110{ }^{\circ} \mathrm{C}$. Finally, the crystallization and template removal were carried out in a muffle furnace at a rate of $1{ }^{\circ} \mathrm{C} / \mathrm{min}$ to $550{ }^{\circ} \mathrm{C}$, holding for $6 \mathrm{~h}$.

$\mathrm{TiO}_{2}$ hydrosol is synthesized by following method. $3 \mathrm{~mL}$ of $\mathrm{Ti}(\mathrm{OBu})_{4}$ was added into $20 \mathrm{~mL}$ of absolute ethanol under modest stir. Then, the uniform solution was added into $30 \mathrm{~mL}$ of $\mathrm{HNO}_{3}(0.04 \mathrm{~mol} / \mathrm{L})$ dropwisely under vigorous stirring to form precipitation. The white suspension was stirring uncovered at $75^{\circ} \mathrm{C}$ for $45 \mathrm{~min}$ to remove alcohol. At last, $60 \mathrm{~mL}$ of $\mathrm{HNO}_{3}(0.04 \mathrm{~mol} / \mathrm{L})$ was added into suspension and the mixed system was aging at $75^{\circ} \mathrm{C}$ for $4 \mathrm{~h}$ to form transparent hydrosol.

Finally, Ti/Cu-MCM-41 was obtained by immersion method. A certain mount of $\mathrm{Cu} / \mathrm{MCM}-41$ was added into 10 $\mathrm{mL}$ of $\mathrm{TiO}_{2}$ hydrosol. Then, the mixed slurry was dried under stirring at $60{ }^{\circ} \mathrm{C}$. The obtained material was calcined at $450{ }^{\circ} \mathrm{C}$ for $3 \mathrm{~h}$ to get final product.

Characterization: The physical or physiochemical properties of washed samples were characterized. The crystalline form of the samples was identified by A Rigaku D/max-2200/ PC XRD instrument $\left(\mathrm{CuK}_{\alpha}\right.$ radiation), operating at $40 \mathrm{kV}$ and $30 \mathrm{~mA}$. The mesoporous phases of the samples were identified at the range of $0.5-8^{\circ}$ with the scanning speed of $0.5^{\circ}$ per minute. Large diffraction angle analysis was conducted at the range of $10-70^{\circ}$ by the same XRD instrument. The BET surface areas and pore size distribution of the samples were measured using a Micromeritics ASAP 2010 system with nitrogen as the adsorption gas. To obtain the morphology of the samples, a high resolution-transmission electron microscopy (JEM100CX, JEOL, Japan) was applied. The coordination environment of $\mathrm{Ti}$ and $\mathrm{Cu}$ in the synthesized catalysts was examined by diffuse reflectance UV-visible spectroscopy with an UV/Vis Spectrometer (Lambda 950, PerkinElmer, USA) in the wavelength range of 200-800 $\mathrm{nm}$. X-ray photoelectron spectroscopy measurements were carried out on a RBD upgraded PHI5000C ESCA system (Perkin Elmer Co. USA).

Photocatalytic test: The gas-phase photodegradation test was similar to Huang's experiment operating in a continuous flow mode ${ }^{14}$. In a typical experiment, formaldehyde was injected into a U-tubular vessel of quartz reactor (U-tube). Before introducing, the initial concentration of formaldehyde was adjusted to $5.0 \mathrm{ppm}$ using $\mathrm{N}_{2} / \mathrm{O}_{2}$ mixture $(\mathrm{v} / \mathrm{v}, 4 / 1)$ in a gas mixer. The inner diameter of the quartz reactor was $6.0 \mathrm{~mm}$ and the height was $250 \mathrm{~mm}$. The reactor, which was filled by $0.5 \mathrm{~g}$ of different catalyst, was illuminated by a couple of UV lamps (8 W, Shanghai Chengguan Illumination Company, China) with maximumlight intensity output at $365 \mathrm{~nm}$ on two sides of the quartz tube. To obtain high intensity of illumination, UV lamps were placed tightly against the quartz reactor. The flow rate $(0.064 \mathrm{~m} / \mathrm{s})$ was controlled by a mass flow controller. The concentration of formaldehyde was analyzed online by gas chromatography (GC) with a flame ionization detector (FID, detection temperature at $200{ }^{\circ} \mathrm{C}$ ) and two columns (carbon molecular sieve and TDX-502). Before the gaseous products went into the FID detector, they must be converted to methane in a methanization converter by $\mathrm{Ni}$ catalyst to ensure a high intensity of signal.

Before photodegradation test, an absorption experiment was also recorded in dark. A certain amount of formaldehyde was injected into the U-tube. In different adsorption experiment, U-tube was filled by same amount of different catalyst. Then, the U-tube was sealed for adsorption. To reduce error, the residual air in the U-tube was pumped out using a vacuum pump before each experiment. Gas samples at different time intervals were withdrawn from a sampling port attached to the side of the vessel. After adsorption experiment, was put under continuous flow mode for some time to get equilibrium. Next, the photodegradation test was directly employed. Studies of the photodegradation test and adsorption of gas-phase formaldehyde were carried out at room temperature $\left(25^{\circ} \mathrm{C}\right)$ by an air-cooling system.

\section{RESULTS AND DISCUSSION}

The phase structures of samples prepared in present work were investigated by XRD and the obtained results were presented in Fig. 1. The small angle XRD patterns of MCM41, Ti-MCM-41, Cu-MCM-41 and Ti/Cu-MCM-41 (Fig.1a) obviously shows that the XRD patterns of MCM-41 and $\mathrm{Cu}-$ MCM-41 include three well-resolved peaks which are indexed as $\left(\begin{array}{lll}1 & 0 & 0\end{array}\right),\left(\begin{array}{lll}1 & 1 & 0\end{array}\right)$ and $\left(\begin{array}{lll}2 & 0 & 0\end{array}\right)$ diffractions, suggesting their typical mesoporous structure ${ }^{15}$. The incorporation of Ti would markedly weaken the orderliness of mesoporous structure, regarding to the decreasing peak intensities. Comparing the XRD patterns of Ti-MCM-41 and Ti/Cu-MCM-41, the (1 $\left.\begin{array}{lll}1 & 0 & 0\end{array}\right)$ peak of Ti/Cu-MCM-41 is more intensive than that of TiMCM-41. It seems the incorporation of $\mathrm{Cu}$ degrade the adverse 
impact of Ti on MCM-41 framework. We supposed that the appearance of pore blocking was occurred on the surface of MCM-41 support attributing to $\mathrm{TiO}_{2}$ aggregate and $\mathrm{Cu}$ could improve the dispersion of $\mathrm{TiO}_{2}$. The large angle X-ray diffraction patterns of four samples shows that each pattern has a broad band at $2 \theta=23^{\circ}$, declaring the existence of amorphous $\mathrm{SiO}_{2}$ (Fig. 1b). Besides, in the patterns of Ti-MCM41 and $\mathrm{Ti} / \mathrm{Cu}-\mathrm{MCM}-41$, one can see that an intensive peak appears at $2 \theta=25.1^{\circ}$, suggesting the formation of the anatase
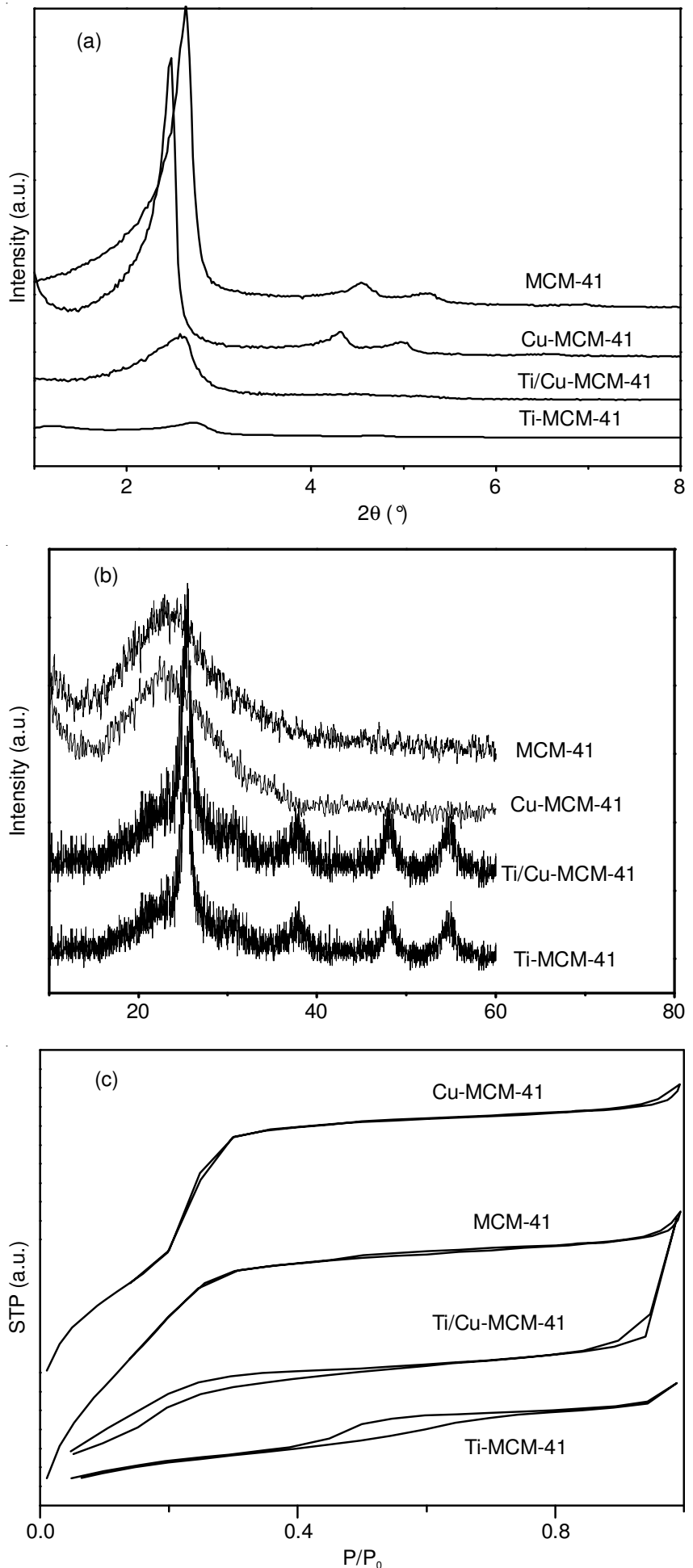

Fig. 1. XRD patterns of synthesized samples: (a) Small angle; (b) Large angle; (b) Nitrogen adsorption isotherms of synthesized samples
$\mathrm{TiO}_{2}$. However, in the patterns of $\mathrm{Cu}-\mathrm{MCM}-41$ and $\mathrm{Ti} / \mathrm{Cu}-$ MCM-41, we did not find the characteristic peak of copper. This result was similar as other research ${ }^{13}$. Copper may be dispersed as nano-sized metallic oxides on the surface of MCM-41 support.

The micromorphologies of synthesized samples can be illustrated by transmission electron microscopy. The TEM images of MCM-41, Ti-MCM-41, Cu-MCM-41 and Ti/CuMCM-41 confirmed that all the samples own a two-dimensional hexagonal mesopores (Fig. 2). The introduction of $\mathrm{Cu}$ and Ti did not affect their uniform structure, corresponding to the results of $\mathrm{X}$-ray diffraction and $\mathrm{N}_{2}$ adsorption isotherm. Moreover, all samples are spherical with particles 300-400 $\mathrm{nm}$ in diameter. This may attribute to the presence of ethanol in the synthesis media. In high concentration ethanol solution, the growth of silicate micelles is driven by global surface tension. Therefore, they are prone to form the shape of a sphere to minimize their surface free energy ${ }^{16}$. None of visible aggregates is detected in Fig. 2c, which means copper oxides are highly dispersed on the surface of catalyst supports. However, some cognizable metal oxides aggregates can be seen in Fig. $2 \mathrm{~b}$ and $2 \mathrm{~d}$. Comparing to Fig. 2c, we considered these aggregates belonged to $\mathrm{TiO}_{2}$. The Ti loading level in this study could not form an indistinguishable metal oxide layer outside MCM-41 support. As a result, XRD analysis also testified the presence of anatase $\mathrm{TiO}_{2}$. In addition, the agglomerate phenomenon of $\mathrm{Ti}$ in Fig. $2 \mathrm{~b}$ is more serious than that of in Fig. 2d. As a result, we observed the weakest intensity of (100) peak in XRD pattern of Ti-MCM-41.

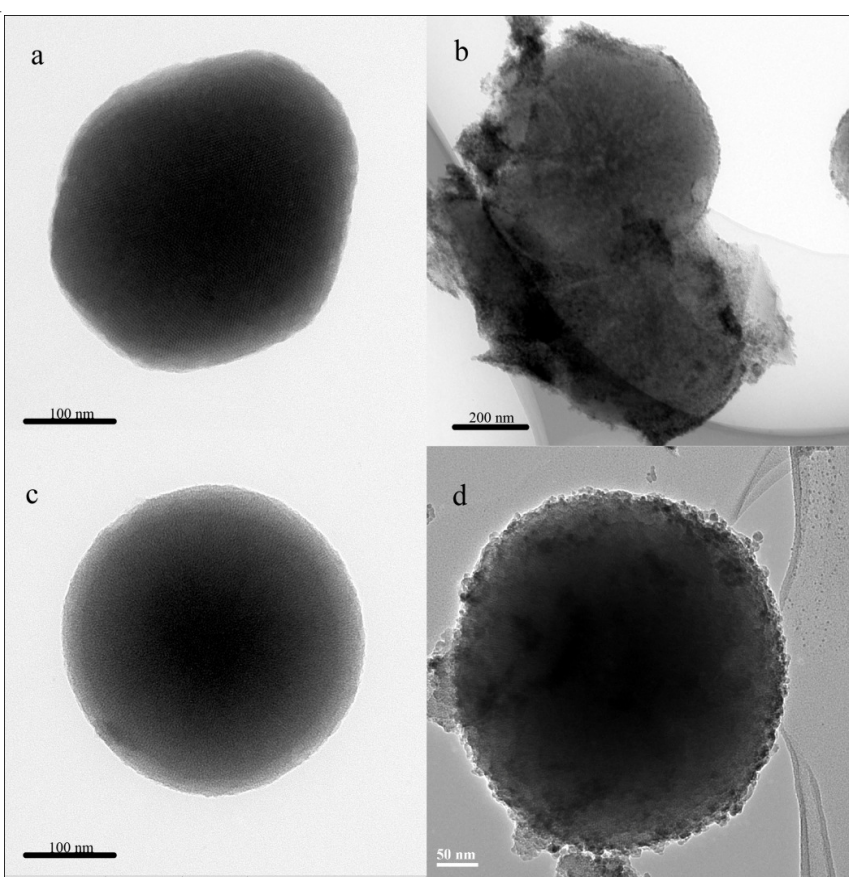

Fig. 2. TEM micrographs of synthesized samples: (a) MCM-41; (b) TiMCM-41; (c) Cu-MCM-41; (d) Ti/Cu-MCM-41

Each sample's nitrogen adsorption-desorption isotherm spectrum fits an isotherm of type IV (Fig. 1c), which is typical for the mesoporous materials ${ }^{15}$. This result was in accord with X-ray diffraction analysis. Noticeably, after metals doping, the inflections at $\mathrm{p} / \mathrm{p}_{0}=0.2-0.3$ in the isotherms became 
smooth, indicating a less ordered mesoporous structure. The isotherms of all samples do not contain a significant hysteresis loop except for Ti/Cu-MCM-41. The appearance of such loop can attribute to the macro pores of interparticles ${ }^{17}$. Besides, the structural and chemical properties of synthesized samples were confirmed by BJH method (Table-1).

\begin{tabular}{lcccc}
\multicolumn{5}{c}{ TABLE-1 } \\
$\begin{array}{l}\text { STRUCTURAL AND CHEMICAL PROPERTIES } \\
\text { OF SYNTHESIZED SAMPLES }\end{array}$ \\
\hline Sample & $\begin{array}{c}\mathrm{S}_{\mathrm{BET}} \\
\left(\mathrm{m}^{2} / \mathrm{g}\right)\end{array}$ & $\begin{array}{c}\mathrm{V}_{\mathrm{p}} \\
\left(\mathrm{cm}^{3} / \mathrm{g}\right)^{\mathrm{a}}\end{array}$ & $\mathrm{p}_{\mathrm{d}}(\AA)^{\mathrm{b}}$ & $\mathrm{d}_{\mathrm{p}}(\mathrm{nm})^{\mathrm{c}}$ \\
\hline $\mathrm{MCM}-41$ & 1545.60 & 0.83 & 20.45 & 2.63 \\
$\mathrm{Cu}-\mathrm{MCM}-41$ & 1402.53 & 0.86 & 23.23 & 2.67 \\
Ti-MCM-41 & 351.76 & 0.33 & 21.65 & 1.85 \\
Ti/Cu-MCM-41 & 726.15 & 0.66 & 16.02 & 1.83 \\
\hline $\mathrm{a}=$ Total pore volume \\
$\mathrm{b}=$ Pore diameter $\left(\mathrm{D}_{\text {BJH }}\right)$ \\
$\mathrm{c}=$ Average pore diameter, calculated by BJH method \\
\hline
\end{tabular}

After Ti incorporation, the BET surface area and total pore volume of Ti/Cu-MCM-41 and Ti-MCM-41 declined a lot due to the blocking effect of $\mathrm{TiO}_{2}$. Corresponding to the obtained results of X-ray diffraction and transmission electron microscopy analysis, Ti/Cu-MCM-41 owned a larger surface area compared to that of Ti-MCM-41.

The UV-visible diffuse reflectance spectra for different samples were presented (Fig. 3). As shown in the figure, after copper-doping, Ti/Cu-MCM-41 exhibited a strong adsorption band ranging from 380 to $570 \mathrm{~nm}$, whereas the absorption edge of Ti-MCM-41 is located at around $380 \mathrm{~nm}$. Definitely, it is $\mathrm{Cu}$ which broadens the available range of Ti-MCM-41. Moreover, in the spectrum of Ti/Cu-MCM-41, another adsorption band between 580 and $800 \mathrm{~nm}$ is also observed. This absorption band can be assigned to the absorption of $\mathrm{Cu}_{2} \mathrm{O}$ and $\mathrm{CuO}$ on the $\mathrm{TiO}_{2}$ surface ${ }^{18}$. It is said that the band between 800 and $500 \mathrm{~nm}$ refers to the $2 \mathrm{E}_{\mathrm{g}}-2 \mathrm{~T}_{2 \mathrm{~g}}$ transitions of $\mathrm{Cu}^{2+}$ as well as to the presence of $\mathrm{Cu}^{+}$clusters reduced from the $\mathrm{Cu}-\mathrm{O}$ matrix $^{19}$

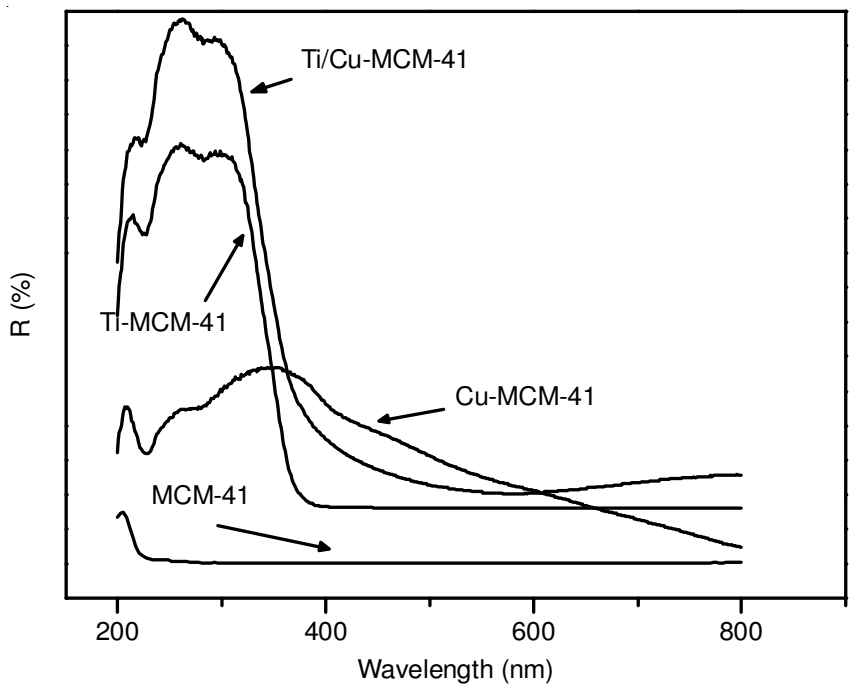

Fig. 3. (a) DRS spectra and for synthesized samples

To discuss the evolution of surface properties of Ti/MCM41 with and without $\mathrm{Cu}$ species, the XPS spectrum of Ti/MCM-
41 and Ti/Cu-MCM-41 were measured (Fig. 4). The Cu2 $\mathrm{p}_{3 / 2}$ XPS peak at $932 \mathrm{eV}$ was observed for Ti/Cu-MCM-41, as shown in Fig. 4a. It was said that the $\mathrm{Cu} 2 \mathrm{p}_{3 / 2}$ XPS peaks at 934 and $935 \mathrm{eV}$ correspond to $\mathrm{Cu}(\mathrm{II})$ in $\mathrm{CuO}$ and $\mathrm{Cu}(\mathrm{OH})_{2}$, respectively, whereas that at $932.5 \mathrm{eV}$ refers to $\mathrm{Cu}(\mathrm{I})$ in $\mathrm{Cu}_{2} \mathrm{O}^{20}$. Thus, Fig. 6a indicates that there are two components of $\mathrm{Cu}$ species, which are attributed to $\mathrm{Cu}_{2} \mathrm{O}(928.9 \mathrm{eV})$ and $\mathrm{CuO}$ (931.1 eV) on the surface of MCM-41. Fig. 4b shows the XPS spectra relating the binding energy of the $\mathrm{Ti} 2 \mathrm{p}$ for two samples. The Ti 2p spectra of Ti/MCM-41 and Ti/Cu-MCM-41 exhibited peaks at 458.6 and $459.0 \mathrm{eV}$, respectively ${ }^{21,22}$, assigned to the typical $\mathrm{Ti}^{4+}$. Obviously, the peaks were shifted to the higher energy side with the incorporation of $\mathrm{Cu}$ in Ti/Cu-MCM-41. This fact suggests that there is an intense interaction between $\mathrm{Ti}^{4+}$ and $\mathrm{Cu}^{2+}$. The Fermi level of $\mathrm{TiO}_{2}$ is higher than that of $\mathrm{Cu}_{2} \mathrm{O}$ and $\mathrm{CuO}^{17}$. When $\mathrm{TiO}_{2}$ intensively mixed with $\mathrm{Cu}$ speies, the electrons transfer from $\mathrm{TiO}_{2}$ to the $\mathrm{Cu}_{2} \mathrm{O}$ and $\mathrm{CuO}$ is expected to occur.
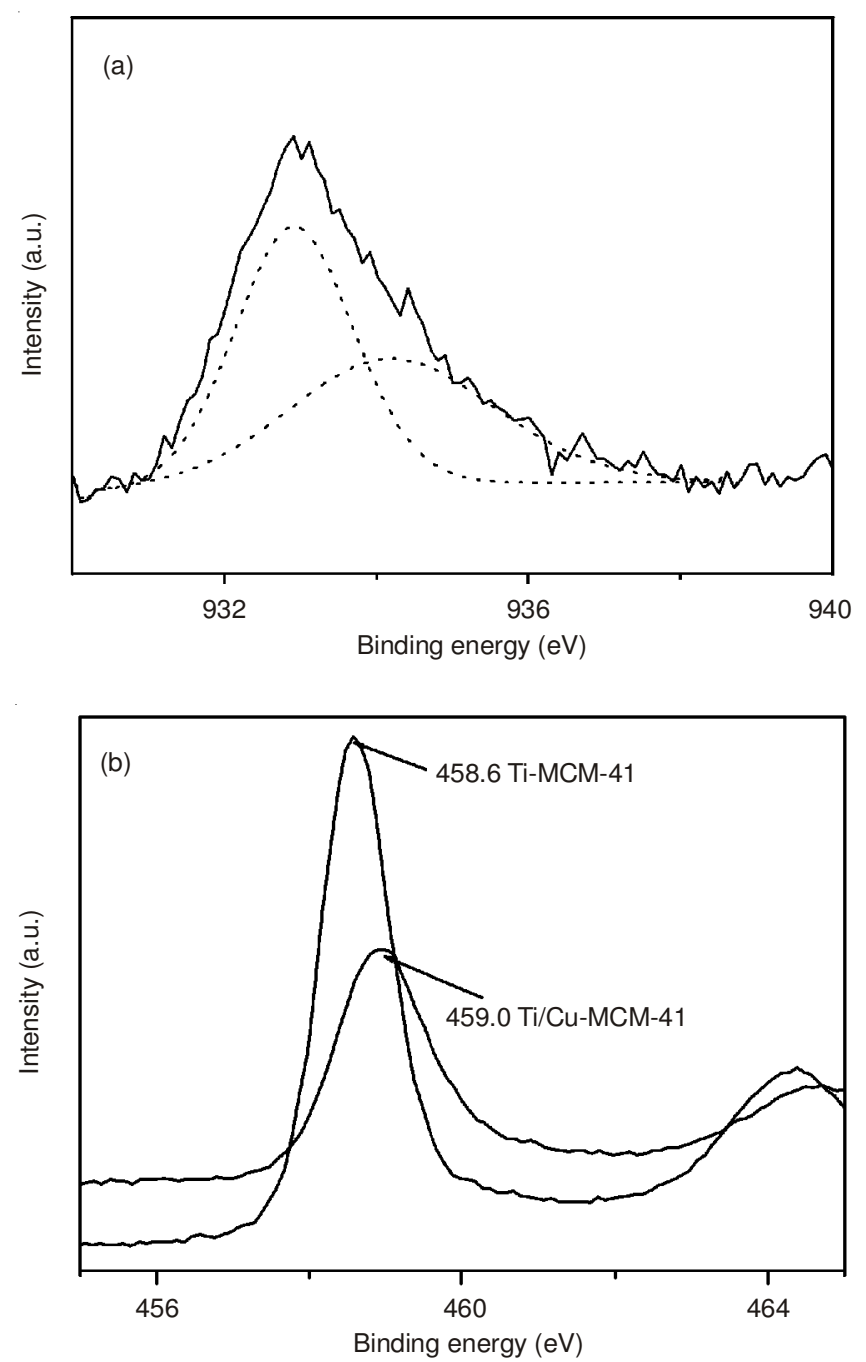

Fig. 4. (a) $\mathrm{Cu} 2 \mathrm{p} 3 / 2 \mathrm{XPS}$ spectrum for Ti/Cu-MCM-41; (b) Ti 2p XPS spectra for Ti-MCM-41 and Ti/Cu-MCM-41

Photocatalytic performance: In this work, an adsorption test of various catalysts was investigated before photocatalytic reaction. MCM-41 possesses the lowest adsorption property in four materials [Fig. 5(a)]. With the increase of metals content 
in catalysts, formaldehyde adsorption capacities increase. This observation can be due to the improved hydrophilism of catalysts. The addition of metals in mesoporous silica increased the concentration of the surface unsaturated metal centers, which would attract more dissociated $\mathrm{H}_{2} \mathrm{O}$ molecules to form surface hydroxyl groups ${ }^{23}$. Consequently, the adsorption property of catalyst towards formaldehyde was elevated with increased hydrophilism. Moreover, to testify the superior adsorption property of Ti-MCM-41 over $\mathrm{TiO}_{2}$ supported on activated carbon $\left(\mathrm{TiO}_{2} / \mathrm{AC}\right)$, another adsorption experiment was performed. $\mathrm{TiO}_{2} / \mathrm{AC}$ was prepared following the same synthesis procedure as Ti-MCM-41 except calcined at $550{ }^{\circ} \mathrm{C}$. The experiment condition was same as MCM-41 adsorption and the result showed that $\mathrm{TiO}_{2} / \mathrm{AC}$ could only get $23 \%$ of formaldehyde removal.

After adsorption test, the mesoporous materials of MCM41, Cu/MCM-41, Ti/MCM-41 and Ti/Cu-MCM-41 were used to compare their respective catalytic activity in formaldehyde degradation [Fig. 5(b)]. In the control experiment, the commercial P25 powder was used. Compared to pure MCM-41, other metals-doped catalysts showed a considerably higher formaldehyde degradation performance. After $4 \mathrm{~h}$ reaction, the degradation performance of each material reached an equilibrium point. Cu-MCM-41 and P25 removed $15 \%$ and $29 \%$ of formaldehyde, respectively. Ti-MCM-41 exhibited a supeior degradation property than $\mathrm{P} 25$, reaching $36 \%$ of formaldehyde removal. Better dispersion of $\mathrm{TiO}_{2}$ on the surface of MCM-41 support made Ti-MCM-41 more effective, comparing with P25. Strikingly, Ti/Cu-MCM-41 achieved $57 \%$ of formaldehyde removal, higher than the removal efficiency summation of Ti/MCM-41 and Cu-MCM-41. The observed higher adsorption ability of Ti/Cu-MCM-41 can explain the enhanced degradation. Besides, incorporated $\mathrm{Cu}$ in $\mathrm{Ti} / \mathrm{Cu}-\mathrm{MCM}-41$ gave the synergistic effect in catalytic reaction. As is observed in Fig. 4, a charge transfer occurred between $\mathrm{TiO}_{2}$ and $\mathrm{Cu}$ species. As a result, doped $\mathrm{Cu}$ forms electronic capture center in film forbidden zone of $\mathrm{TiO}_{2}$ to hinder the combination of $\mathrm{h}^{+}$and $\mathrm{e}^{-}$ , which enables $\mathrm{e}^{-}$to absorb less energy than that of forbidden band and to jump from valence band to conduction band. Moreover, according to X-ray diffraction and transmission electron microscopy analyses, $\mathrm{Cu}$ species could enhance the dispersion of $\mathrm{TiO}_{2}$ on the surface of catalyst.

Furthermore, the visible-light catalytic abilities of various samples were investigated, following photocatalytic experiment [Fig. 5(c)]. The visible-light source was offered by two cool day light lamps of each $25 \mathrm{~W}$ (Philips, the Netherlands), emitting a visible radiation in the range of 400-700 $\mathrm{nm}$. As is shown in Fig. 5(c), the degradation efficiency of MCM-41 and Ti/MCM-41 is negligible. Similar tendency could be observed for P25. On the contrary, Cu-MCM-41 achieved $7 \%$ of formaldehyde degradation. Noticeably, Ti/Cu-MCM-41 removed $18 \%$ of formaldehyde, indicating the promotion effect of $\mathrm{Cu}$ on visible-light catalytic performance of Ti. This result just corresponds with DRS analysis.

\section{Conclusion}

A bimetallic photochemical catalyst (Ti/Cu-MCM-41) and other analogous catalysts were obtained in two-steps synthesis procedure. The resulting material possessed a typical meso-
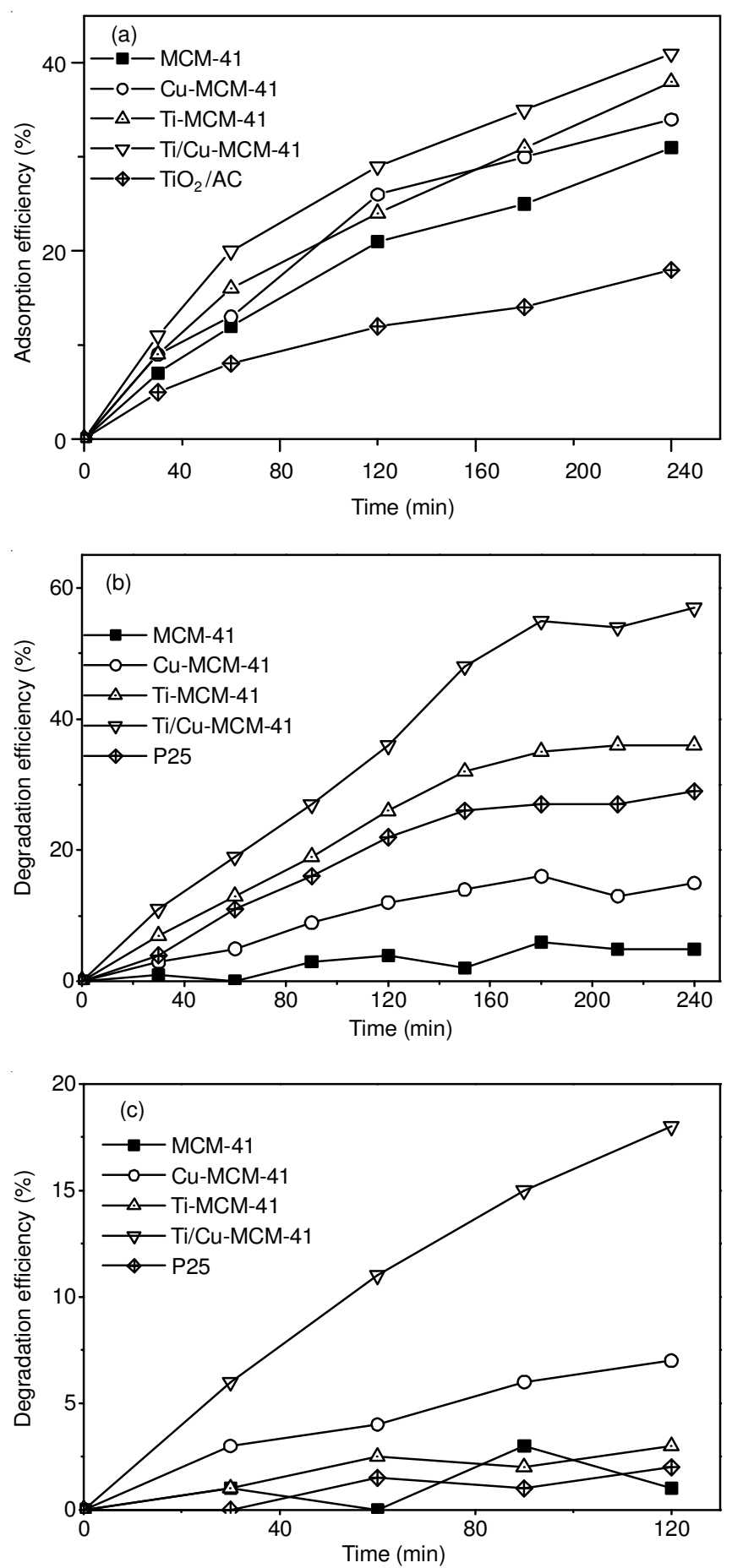

Fig. 5. (a) Adsorption capacities of catalysts for formaldehyde; (b) Photocatalytic degradation of formaldehyde under UV irradiation; (c) Photocatalytic degradation of formaldehyde under visiblelight irradiation

porous structure. At the end of synthesis, $\mathrm{Cu}$ species were highly dispersed on the MCM-41 support. Therefore, they could not be detected by X-ray diffraction and transmission electron microscopy analyses. However, $\mathrm{TiO}_{2}$ was recognizable regarding to its loading value. $\mathrm{UV}$-Visible results demonstrated that $\mathrm{Cu}$ existed as $\mathrm{Cu}_{2} \mathrm{O}$ and $\mathrm{CuO}$ on the $\mathrm{TiO}_{2}$ surface so it could act as electronic capture center to suppress the charge recombination of the electrons and holes. Consequently, by XPS analysis, an intense interaction between $\mathrm{Ti}^{4+}$ and $\mathrm{Cu}^{2+}$ was observed. As a result, Ti/Cu-MCM-41 showed the most 
efficient in photocatalytic degradation of formaldehyde. Besides, it also demonstrated best adsorption property of formaldehyde due to its strongest hydrophilism. Additionally, the incorporation of copper also improved its visible-light photo catalytic performance.

\section{ACKNOWLEDGEMENTS}

This work was supported by the Zhejiang Provincial Science and Technology Project of China (Grant No. 2011C31041)

\section{REFERENCES}

1. X.F. Tang, Y.G. Li, X.M. Huang, Y. Xu, H. Zhu, J. Wang and W. Shen, Appl. Catal. B, 62, 265 (2006).

2. T.N. Obee and R.T. Brown, Environ. Sci. Technol., 29, 1223 (1999).

3. C. Young, T.M. Lim, K. Chiang, J. Scott and R. Amal, Appl. Catal., B, 78, 1 (2008).

4. W.K. Jo and K.H. Park, Chemosphere, 57, 555 (2004).

5. S.-S. Kim, D.-H. Kang, D.-H. Choi, M.-S. Yeo and K.-W. Kim, Build. Environ., 43, 320 (2008).

6. J.H. Mo, Y.P. Zhang, R. Yang and Q. Xu, Build. Environ., 43, 238 (2008).

7. Y.X. Sun, Fang, D.P. Wyon, A. Wisthaler, L. Lagercrantz and P. StrømTejsen, Build. Environ., 43, 258 (2008).
8. X.S. Zhao, G.Q. Lu and X. Hu, Micropor. Mesopor. Mater., 41, 37 (2000).

9. E.W. Shin, H.S. Choi, T.-D. Nguyen-Phan, J.S. Chung and E.J. Kim, J. Ind. Eng. Chem., 14, 510 (2008).

10. H.H. Yiu, P.A. Wright and N.P. Botting, J. Mol. Catal. B, 15, 81 (2001).

11. A. Sayari and S. Hamoudi, Chem. Mater., 13, 3151 (2001).

12. L.Q. Fu, L.S. Yang, T. Wang and Q.L. Zhang, Appl. Mech. Mater., 55, 1648 (2011).

13. M. Xia, M.C. Long, Y.D. Yang, C. Chen, W. Cai and B. Zhou, Appl. Catal. B, 110, 118 (2011).

14. X. Huang, W.J. Shi, J. Yuan, J. Shi, Z. Jiang and W. Shangguan, Environ. Technol., 32, 307 (2011).

15. S.C. Laha and R.Kumar, Micropor. Mesopor. Mater., 53, 163 (2002).

16. S.Q. Liu, P. Cool, O. Collart, P. Van Der Voort, E.F. Vansant, O.I. Lebedev, G. Van Tendeloo and M. Jiang, J. Phys. Chem. B, 107, 10405 (2003).

17. K.S.W. Sing, D.H. Everett and R.A.W. Haul, Pure Appl. Chem., 57, 603 (1985).

18. B.F. Xin, P. Wang, D. Ding, J. Liu, Z. Ren and H. Fu, Appl. Surf. Sci., 254, 2569 (2008)

19. G. Colón, M. Maicu, and M.C. Hidalgo, Appl. Catal., B, 67, 41 (2006).

20. H. Irie, K. Kamiya, T. Shibanuma, S. Miura, D.A. Tryk, T. Yokoyama and K. Hashimoto, J. Phys. Chem. C, 113, 10761 (2009).

21. J. Ren, Z. Li and S.S. Liu, Catal. Lett., 124, 185 (2008).

22. X.T. Gao and S.E.I. Wachs, Catal. Today, 51, 233 (1999).

23. H. Tamura, K. Mita, A. Tanaka and M. Ito, J. Colloid Interf. Sci., 243, 202 (2001) 